\title{
Opinion on Patrick Suppes 1957 Introduction to Logic
}

\author{
Walter Gomide \\ waltergomide@yahoo.com \\ Department of Philosophy \\ Institute of Humanities and Social Sciences \\ Federal University of Mato Grosso \\ Brazil \\ 78060-900
}

Submitted: 6 June 2021

Revised: 25 June 2021

\begin{abstract}
We give an opinion on the merits of Patrick Suppes' 1957 book, "Introduction to Logic."
\end{abstract}

\section{Opinion}

The book "Introduction to Logic" by Patrick Suppes, published in 1957 [4] [1], can be considered one of the best beginners' logic manuals ever conceived. His presentation of the definitions and fundamental concepts of logic, which covers the first seven sections of Part I, is both clear and particularly didactic: several examples and very clear explanations are presented in order to introduce the beginning student to logic without excesses of unnecessary technicalities.

Also in Part I of the book, Section 8, devoted to definition theory, is noteworthy. In this section, the problem of division by zero is presented in formalised arithmetic, a topic of special interest to students of transreal numbers or transmathematics as a whole. Patrick Suppes' approach to the introduction of division by zero in formalised arithmetic, as it could not fail to be due to the very formal character of the arithmetic we are dealing with here, is eminently syntactic. Suppes presents several possibilities for the definition of fractions with denominator zero, in such a way that in all attempts there is an impossibility of syntactic generation of contradictions. Very valid, very interesting. However, the introduction of division by zero in formalised arithmetic becomes more interesting if, in addition to the requirement that such a procedure does not cause contradictions (which guarantees the existence of a model of the arithmetic), 
we also introduce the philosophical condition that this division operation, now of a total character, in some way refers to or is interpreted as what is expected geometrically or in the domain of the analysis of fractions with zero denominator, namely, the reference to the infinity or to the indeterminate, in such way that, in the first case, we have fractions of type $c / 0$, with a nonzero $c$, and in the second case, we have the fraction $0 / 0$.

That philosophical concern or care seems to be absent from the possibilities offered by Suppes for division by zero. However, they are very present in the Transreal Numbers [2] [3]; and, for this reason, I believe that division by zero, as introduced in the Transreal Numbers, satisfies both the demand for a calculus that is simultaneously consistent and with an expected semantics from the philosophical point of view: fractions with denominators equal to zero must totalise arithmetic from the admission that their referents are the infinity, in the sense of the largest existing number, or the indeterminate: a number that cannot be compared with the others by a relation of order.

Part II of Suppes' book consists of a very pedagogical or didactic presentation of an intuitive set theory. In this presentation, it is worth mentioning Part II, Section 12, in which the notion of "set theoretical predicates" appears. Basically, a "set theoretical predicate" is a translation into intuitive theory of sets of concepts or predicates of well-established scientific theories. With this, the foundation or the first step for the presentation of scientific theories axiomatised in set theory is achieved. Noteworthy is the way in which, through the concept of the set- theoretical predicate, Suppes presents an axiomatised theory of probability and a theory of Newtonian mechanics "mirrored" in set theory.

In conclusion, it can be said that Suppes' book is an excellent initial presentation of logic, as well as set theory, due to its clarity and philosophical way of approaching the essential aspects of logic and set theory. In the latter, Suppes finds a fundamental tool for the axiomatisation of scientific theories in such a way that they acquire an exposition that can remove or

minimise any conceptual inaccuracies that non-axiomatised expositions of scientific theories may have.

\section{References}

[1] J.A.D.W. Anderson and J.A. Bergstra. Review of suppes 1957 proposals for division by zero. Transmathematica, pages 1-10, 8 June 2021. https://doi.org/10.36285/tm.53

[2] J.A.D.W. Anderson, N. Völker, and A.A. Adams. Perspex machine viii: Axioms of transreal arithmetic. In Longin Jan Lateki, David M. Mount, and Angela Y. Wu, editors, Vision Geometry XV, volume 6499 of Proceedings of SPIE, pages 2.1-2.12, 2007. https://www.doi.org/ $10.1117 / 12.698153$

[3] T.S. dos Reis, W.Gomide, and J.A.D.W. Anderson. Construction of the transreal numbers and algebraic transfields. IAENG International Journal of Applied Mathematics, 46(1):11-23, 2016. http: //www . iaeng.org/IJAM/issues_v46/issue_1/IJAM_46_1_03.pdf 
[4] P. Suppes. Introduction to Logic. Van Nostrand Reinhold Company, 1957. http://web.mit.edu/gleitz/www/Introduction $\% 20$ to\% 20Logic $\% 20-\% 20$ P. $\% 20$ Suppes $\% 20$ (1957) \%20WW.pdf 